Characterization of a helicon plasma source in low diverging magnetic fields

This article has been downloaded from IOPscience. Please scroll down to see the full text article.

2011 J. Phys. D: Appl. Phys. 44055202

(http://iopscience.iop.org/0022-3727/44/5/055202)

View the table of contents for this issue, or go to the journal homepage for more

Download details:

IP Address: 130.56.71.133

The article was downloaded on 17/01/2011 at 11:26

Please note that terms and conditions apply. 


\title{
Characterization of a helicon plasma source in low diverging magnetic fields
}

\author{
T Lafleur, C Charles and R W Boswell \\ Space Plasma, Power and Propulsion Group, Research School of Physics and Engineering, \\ The Australian National University, Canberra ACT 0200, Australia \\ E-mail: trevor.lafleur@anu.edu.au
}

Received 29 August 2010, in final form 24 November 2010

Published 17 January 2011

Online at stacks.iop.org/JPhysD/44/055202

\begin{abstract}
A detailed experimental characterization has been performed of a helicon mode in low nonuniform magnetic fields $\left(B_{0}<5 \mathrm{mT}\right)$ for pressures between $0.04 \mathrm{~Pa}<p_{0}<0.4 \mathrm{~Pa}$ and rf powers between $50 \mathrm{~W}<P_{0}<400 \mathrm{~W}$, using a number of electrostatic probes as well as circuit measurements of the antenna and matching network system. The helicon mode is produced over a narrow range of magnetic field values, where a distinct density peak is formed that becomes broader (and higher) as the power or pressure is increased. The density peak is found to shift to larger magnetic fields for increasing powers or pressures, giving an almost linear relationship between the maximum density and the magnetic field at this maximum density, in agreement with helicon dispersion theory. The density within the peak $\left(>10^{17} \mathrm{~m}^{-3}\right)$ is more than an order of magnitude larger than that before or after, and is associated with a corresponding peak in the measured antenna resistance, thus showing a larger percentage of the input power is deposited within the plasma.
\end{abstract}

\section{Introduction}

Helicon waves, first employed by Boswell [1,2], have become synonymous with rf plasmas, and still remain one of the most efficient methods for producing high density plasmas, often able to produce densities an order of magnitude larger than capacitively or inductively coupled systems of similar powers inputs. Helicon waves are right-hand polarized electromagnetic waves that propagate in magnetized plasmas, and for typical laboratory conditions the plasma refractive index is approximately $100[3,4]$. Conventional helicon systems typically have magnetic fields with strengths of the order of 10-100 mT. Helicon systems are often quite versatile, as depending on the power input and applied magnetic field, they can operate in all three coupling regimes with the plasma (capacitive, inductive and wave mode) [5]. In order to sustain a helicon mode with a given wavelength (which is often determined by some characteristic length, such as that of the antenna), a certain density and magnetic field are required. Simple helicon theory tells us that the required density is then proportional to the applied magnetic field [3]. For a given magnetic field (or power), as the system power (or magnetic field) is increased, discrete mode jumps from capacitive to inductive, to wave mode (helicon) are often seen [5]. However, at low magnetic fields $(<5 \mathrm{mT})$, a direct capacitive to wave mode transition sometimes occurs [6], which typically produces a local density peak around a narrow range of magnetic field values. Since the applied magnetic field is low, a low plasma density is needed to sustain the mode, and consequently, these low-field helicons can be produced with fairly low power inputs. These factors have made lowfield helicons potentially attractive for plasma processing and propulsion applications, since they can in principle result in lower mass and power requirements [7-9].

Low-field helicons have been observed in a number of experiments [10-17], both with different working gases and applied frequencies [14], as well as a range of different rf antennas [12,13,17]. A local peak in the measured antenna resistance is also often observed [14, 17], showing that power is coupled more efficiently into the plasma, and explaining the increased density for the same apparent power input. While very little theoretical work has been done to reproduce these density peaks, computational studies [18, 19] have shown resistance peaks can be formed at low magnetic fields. This numerical work typically calculates the plasma resistance for a given antenna geometry under assumed plasma density 
profiles (so far only in the radial direction, with uniform axial profiles assumed). Results of the numerical work suggests that wave reflection at axial boundaries produces low-field peaks for $m=0$ antennas [18,19], while peaks can be produced with $m=1$ antennas regardless of reflections [19].

While the majority of work thus far on low-field helicons has typically focused on the use of uniform magnetic fields, nonuniform or diverging magnetic fields are of interest for propulsion applications, especially since ion beam formation often relies on plasma expansion due to the applied magnetic field [17, 20,21]. Although some work with diverging fields has been performed, notably by Chen [7-9], the characterization work has typically focused on radial density profiles, or optimization of system geometry through the use of computational codes (although a detailed radial characterization was performed in [8], low-field density peaks were not observed for the majority of measurements taken). Recent results in diverging fields have shown that additional effects (which are absent from uniform fields, and have not been reported before for the studies using nonuniform fields described above) can be present in nonuniform magnetic fields $[17,22]$. In [17] a direct capacitive to wave mode transition was observed, together with the formation of an expanding plasma, wherein which an ion beam was observed in the downstream region. Further experiments [22] showed that this ion beam, together with the plasma density and plasma potential could be controlled by altering the helicon wave propagation through small changes in the magnitude (and geometry) of the magnetic field near the plasma source exit. Detailed experimental studies have not yet been performed in these systems, and it is the aim of this paper to address this. In this investigation, low-field helicon modes are produced with a single, short solenoid, so that the magnetic field resembles that of a magnetic dipole, and thus is significantly nonuniform. A detailed experimental characterization of the plasma source region is then conducted, using electrostatic probe measurements within the plasma, and electrical measurements in the matching network/antenna circuit.

\section{Apparatus}

Experiments are performed in the Piglet helicon reactor that has been described previously [17,22], and is shown in figure 1 . The reactor consists of a $20 \mathrm{~cm}$ long Pyrex source tube connected to a $28.8 \mathrm{~cm}$ aluminium diffusion chamber. The opposite end of the source tube is closed with a grounded metal grid, to which a rotary/turbomolecular pump system is connected. The source tube radius is $6.8 \mathrm{~cm}$ while the diffusion chamber radius is $16 \mathrm{~cm}$. Plasma is created using an rf double-saddle field antenna that surrounds the source tube. The antenna is connected to a $\pi$ matching network, a schematic of which is shown in figure 2, and an rf power generator operated at $13.56 \mathrm{MHz}$. The matching network consists of two tuneable high voltage vacuum capacitors $\left(C_{\text {load }}\right.$ and $C_{\text {tune }}$ ) used to adjust the system impedance and hence match the antenna impedance to the $50 \Omega$ output impedance of the power generator. A directional coupler (not shown in

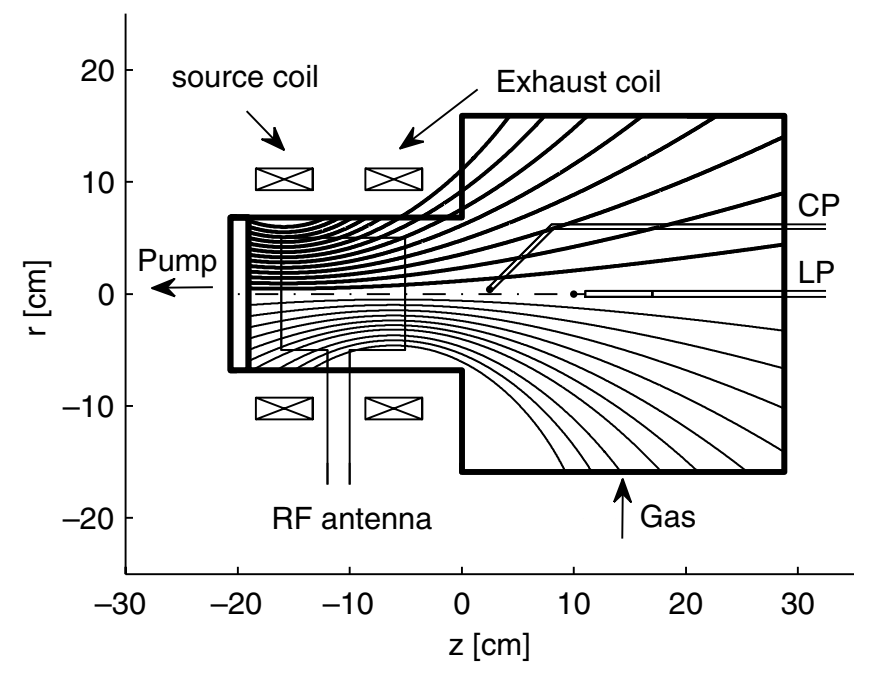

Figure 1. Schematic of the Piglet helicon reactor, including source region $(z<0 \mathrm{~cm})$, diffusion chamber $(z>0 \mathrm{~cm})$ and magnetic field coils. The magnetic field lines for the source coil (bold solid lines; exhaust coil off) are shown above the central axis, while the field lines for the exhaust coil (solid lines; source coil off) are shown below the central axis.

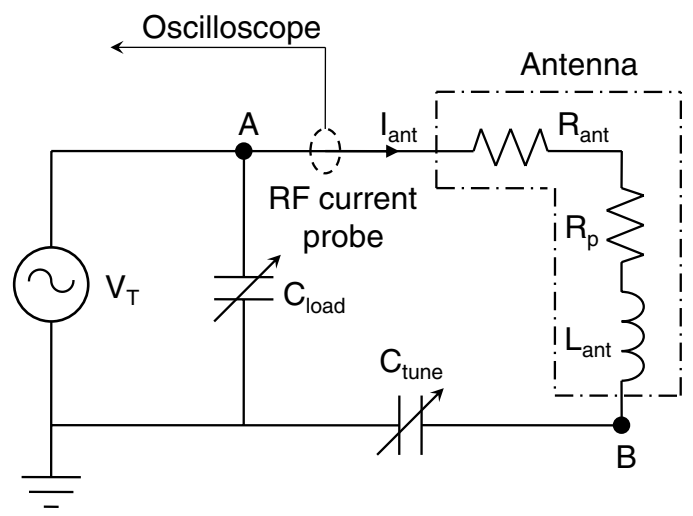

Figure 2. Schematic of the antenna and matching network. The current in the antenna, $I_{\mathrm{ant}}$, is measured with the rf current probe indicated. High voltage probe measurements are made at points marked A and B. $R_{\text {ant }}$ represents the external circuit and antenna resistance, while $R_{\mathrm{p}}$ represents the resistance of the plasma as seen by the antenna.

figure 2) placed between the matchbox and rf generator is used to monitor the forward and reverse power to the matching network. A pair of magnetic field coils, each consisting of approximately 500 turns, surrounds the rf antenna, with the coil closest to the pump termed the source coil, while the other coil is termed the exhaust coil (see figure 1). Argon gas is used for all experiments in this investigation, and is fed into the reactor via a port in the side of the diffusion chamber. The reactor base pressure $(<0.3 \mathrm{mPa})$ is measured with an ion gauge, while a baratron pressure gauge is used to measure the operating pressure. Both pressure gauges are attached to the back plate of the diffusion chamber. In this investigation, diagnostic probes are inserted through ports in the back plate of the diffusion chamber, and can translate axially while still maintaining vacuum integrity. 


\section{Diagnostics}

A Langmuir probe (LP), operated in ion saturation mode, is used to measure the plasma density. The probe consists of a small nickel disc $2 \mathrm{~mm}$ in diameter, which is supported by a hollow ceramic tube. The ceramic tube is then connected to a metal shaft, which passes through a vacuum feedthrough connected to the back plate of the diffusion chamber. The nickel disc is orientated perpendicularly to Piglet's central axis (and hence perpendicularly to the on-axis magnetic field) and is biased at $-45 \mathrm{~V}$. The current collected by the LP is determined from the voltage drop across a $1 \mathrm{k} \Omega$ sense resistor. An rf compensated Langmuir probe $(\mathrm{CP})$ is used to establish the electron temperature (which is used to determine the plasma density from the measured LP current). The $\mathrm{CP}$ has previously been described [23], and in brief, consists of a group of resonant inductors that filter out the fundamental $(13.56 \mathrm{MHz})$ and first harmonic $(27.12 \mathrm{MHz})$ frequencies of the collected current. The probe is connected to a standard sweeping circuit and the current collected is measured from the voltage drop across a $100 \Omega$ sense resistor. This voltage is fed into an isolation amplifier which is connected to a series of two analogue differentiators. The second derivative of the collected current with respect to the bias voltage can be shown to be proportional to the electron energy probability function (EEPF) [24]. The electron temperature is then related to the slope of the natural logarithm of the EEPF. The rf current in the antenna, $I_{\mathrm{rf}}$, is measured using an rf current probe (see figure 2) that surrounds one of the electrical feedthroughs of the antenna. The output of the calibrated probe is connected to a $50 \Omega$ feedthrough terminator and a digital oscilloscope. A high voltage probe is used to measure the rf voltage at different locations within the matching network and antenna, as shown by the points marked A and B in figure 2.

\section{Results}

\subsection{Characterization of low-field density peaks}

Low-field density peaks have previously been observed in the present reactor when operating with just the source coil (exhaust coil off; see figure 1), at an input power of $250 \mathrm{~W}$ and gas pressures of 0.04 and $0.08 \mathrm{~Pa}[17,22]$. We extend this by investigating a larger range of powers $\left(50 \mathrm{~W}<P_{0}<400 \mathrm{~W}\right)$ and pressures $\left(0.04 \mathrm{~Pa}<p_{0}<0.4 \mathrm{~Pa}\right)$. Figure 3(a) shows the plasma density within the source region $(z=-10 \mathrm{~cm})$ as a function of the maximum applied magnetic field, for a number of different input powers. For these measurements, the magnetic field was ramped up from 0 to $16 \mathrm{mT}$ (we consider the case of ramping the magnetic field down later). As seen from the figure, at very low powers $(<100 \mathrm{~W})$ a very small density peak is present at around $B_{0} \approx 1.5 \mathrm{mT}$. As the magnetic field is further increased, the density drops, before again rising monotonically at around $B_{0}>4 \mathrm{mT}$. We note that for these larger magnetic fields (at these low powers), the maximum density is larger than the maximum present at the low-field peak. As the power is increased, we observe that the density peak gets higher (and broader), and that the density is now
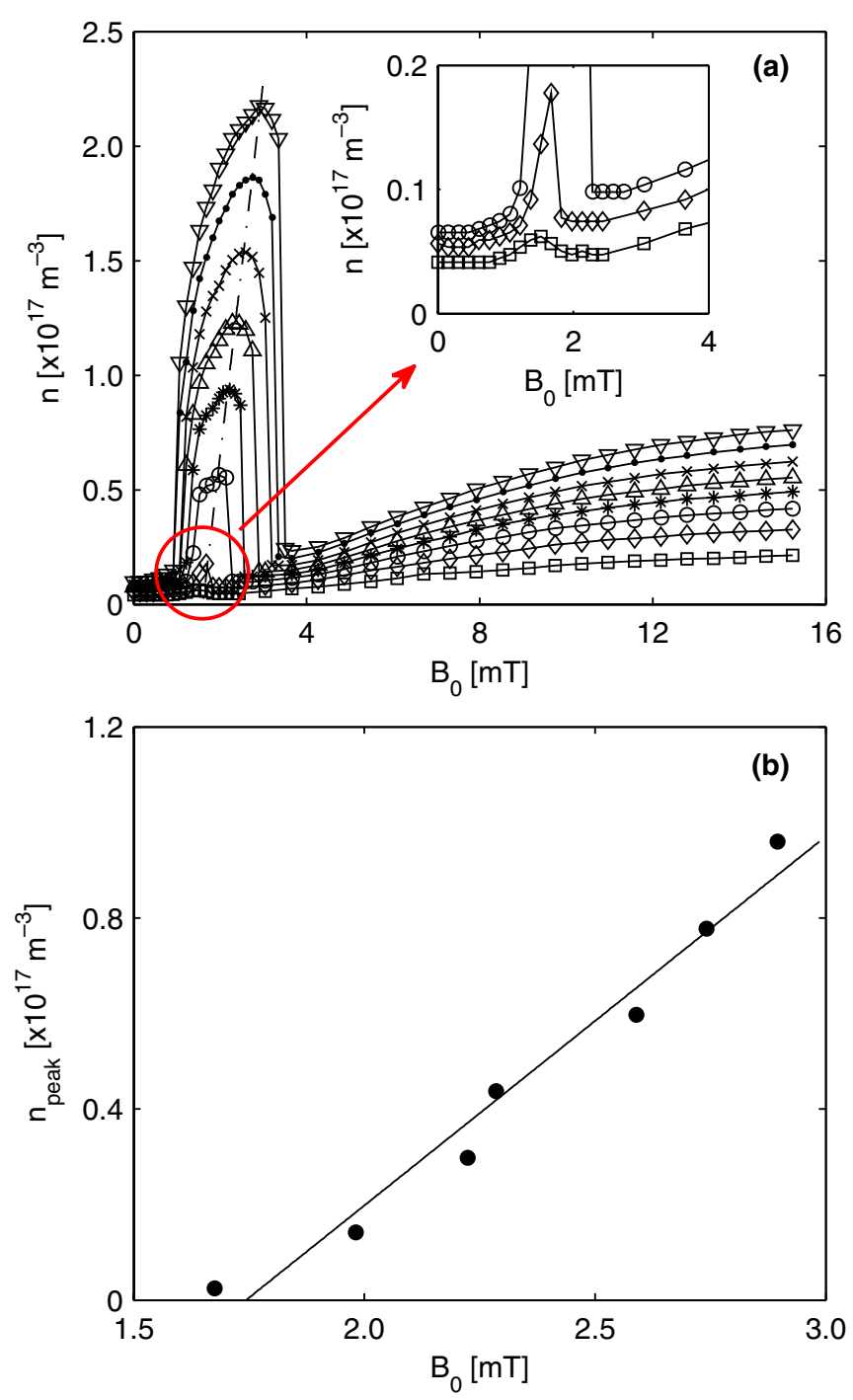

Figure 3. (a) Plasma density within the source region $(z=-10 \mathrm{~cm})$ as a function of the maximum applied magnetic field (as the source coil is varied, with the exhaust coil off), at a number of rf powers; $50 \mathrm{~W}$ (open squares), $100 \mathrm{~W}$ (open diamonds), $150 \mathrm{~W}$ (open circles), $200 \mathrm{~W}$ (stars), $250 \mathrm{~W}$ (open triangles), $300 \mathrm{~W}$ (crosses), $350 \mathrm{~W}$ (dots), $400 \mathrm{~W}$ (open upside-down triangles). The straight dashed line illustrates that the peak density is linearly related to the peak magnetic field as the power is increased. The gas pressures is $0.04 \mathrm{~Pa}$. The inset figure shows a magnified view of the low-power density peaks. (b) Peak density (corrected for sheath area effects of the LP) as a function of peak magnetic field. The solid line is a best-fit linear function, indicating the proportionality identified in $(a)$.

(This figure is in colour only in the electronic version)

larger than that for $B_{0}>4 \mathrm{mT}$, by a factor of almost 3 . At these higher powers, the peak density of around (1-2) $\times 10^{17} \mathrm{~m}^{-3}$ is almost 10 times larger than that occurring just before or after the peaks. It is interesting to note that the maximum value of the peak shifts to larger magnetic fields as the power is increased. The dispersion relation for helicon waves (which assumes a uniform magnetic field and plasma density, and is obtained by including electron inertia and the Hall current term in the generalized Ohm's law [3]), including the effects of finite electron mass (which becomes important at low magnetic 
fields), can be given by $[3,25]$

$$
k_{z} k-k^{2} \frac{\omega}{\omega_{\mathrm{ce}}}=\frac{q \mu_{0} n_{0} \omega}{B_{0}},
$$

where $k=\sqrt{k_{z}^{2}+k_{\perp}^{2}}$ is the total wavenumber, $k_{z}$ and $k_{\perp}$ are the parallel and perpendicular wavenumber components, $\omega$ is the wave angular frequency, $\omega_{\text {ce }}=q B_{0} / m$ is the electron cyclotron frequency, $q$ and $m$ are the electron charge and mass, respectively, $\mu_{0}$ is the permeability of free space and $n_{0}$ is the plasma density. For a given $k_{z}, n_{0}$ and $B_{0}$, equation (1) has in general two solutions for $k_{\perp}\left(k_{\perp_{1}}\right.$ and $k_{\perp_{2}}$, with $\left.k_{\perp_{1}}<k_{\perp_{2}}\right)$ for $\omega<\omega_{\text {ce }} / 2$. The smaller of these $\left(k_{\perp_{1}}\right)$ is generally referred to as the helicon mode, while the larger $\left(k_{\perp_{2}}\right)$ is known as the Trivelpiece-Gould (TG) mode. Note, however, that both solutions satisfy the same dispersion relation. Equation (1) describes only the dispersion relation of waves within the plasma, and says nothing about how the plasma is formed or reaches the density it has. From power balance considerations [24], the density in wave-sustained systems is proportional to the power absorbed by the plasma. Thus as the power input increases we would expect that the plasma density also increases. If the wavenumbers $k_{z}$ and $k_{\perp}$ in equation (1) were constant, then as the density increases this dispersion relation can only continue to be satisfied if the magnetic field changes as well. By rearranging equation (1) we can show that for a given wavenumber, the required magnetic field is a linear function of the plasma density:

$$
n_{0}=c B_{0}-d,
$$

where $c=k_{z} k / q \mu_{0} \omega$, and $d=m k^{2} / q^{2} \mu_{0}$. Double-saddle field antennas (as are used here) are known to most strongly excite waves with a wavelength equal to twice the antenna length [2, 3, 24] (although are not limited to exciting waves of this wavelength only). If we assume this to be true here so that the wavenumber is approximately constant, then as the power input to Piglet increases, we would expect the density to increase, and thus from equation (2) that the magnetic field at the density peak should also increase. The dashed line in figure 3(a) shows that the density peaks do indeed appear to show a linear variation consistent with equation (2). Using standard Langmuir probe analysis methods, the densities in figure 3(a) were found from

$$
I_{\mathrm{sat}}=0.61 q n A u_{\mathrm{B}}
$$

where $I_{\text {sat }}$ is the ion saturation current measured with the LP, $n$ is the plasma density in $\mathrm{m}^{-3}, A$ is the probe area, $u_{\mathrm{B}}=\sqrt{q T_{\mathrm{e}} / M}$, with $M$ the ion mass and $T_{\mathrm{e}}$ the electron temperature in electron volts. As mentioned in section 3, the electron temperature is found from the slope of the measured EEPF. The EEPFs here have a bi-Maxwellian shape, with two distinct electron populations (referred to as the bulk and tail populations, respectively) separated at an energy break close to the plasma potential. These EEPFs are similar to those reported before in a similar reactor $[23,26]$. The plasma densities are found using the temperature of the bulk electron population of the EEPFs, which has a temperature of $8.5 \mathrm{eV}$ at $0.04 \mathrm{~Pa}$, decreasing to about $4 \mathrm{eV}$ at $0.4 \mathrm{~Pa}$. For the density estimates in figure 3(a), edge effects of the LP were not accounted for. Sheridan $[27,28]$ has recently performed detailed simulation work on edge effects and sheath expansion around a disc LP, and established empirical relationships to determine the sheath area as a function of probe bias and disc radius. He performed 2D hybrid-PIC simulations to determine the collecting area of a disc Langmuir probe operated in ion saturation mode (such as is used here). The simulations assume cold ions, and ignore both the structure supporting the disc, and magnetic field effects. Lack of some of these effects was thought to produce an over estimation of the sheath area by less than $15 \%$ [27]. Experimental confirmation of Sheridan's theory has recently been conducted [29]. Sheridan [27] has provided a simple method to calculate the sheath area. By fitting a power law to the simulation data, the sheath area around a LP disc, $A_{\mathrm{s}}$, can be found as a function of the probe radius $r_{\mathrm{p}}$, bias voltage $V_{\text {bias }}$, plasma potential $V_{\mathrm{p}}$, electron temperature $T_{\mathrm{e}}$ and plasma density $n$. The current, $I_{\text {sat }}$, collected by a LP in ion saturation mode is then

$$
I=\kappa q A_{\mathrm{s}} n u_{\mathrm{B}},
$$

where $\kappa \approx 0.55$ (a dimensionless constant) following Sheridan's suggestion [27]. Since $A_{\mathrm{s}}$ now depends on the density, equation (4) must be solved iteratively to find the actual plasma density. Using this approach we calculate the corrected densities for the points located at the peaks in figure 3(a). These corrected densities, $n_{\text {peak }}$, are then plotted in figure $3(b)$ as a function of the magnetic field at this peak density, $B_{\text {peak }}$. The solid line is a best-fit linear function clearly showing the strong linear dependence between the density and magnetic field, this despite the nonuniformities present in the magnetic field (since it is diverging) and plasma density $[17,22]$ (see later). This best-fit function gives $n_{\text {peak }}=$ $0.77 B_{\text {peak }}-1.35$ with $n_{\text {peak }}$ and $B_{\text {peak }}$ in units of $\left(1 \times 10^{17} \mathrm{~m}^{-3}\right)$ and $(m T)$, respectively.

Previous measurements [22] in the present system for a pressure of $0.08 \mathrm{~Pa}$ and input power of $250 \mathrm{~W}$ have estimated the axial wavelength to be approximately $z \approx 0.2 \mathrm{~m}$ (this then gives the axial wavenumber from $k_{z}=2 \pi / \lambda_{z}$ ), and the perpendicular wavenumber to be $k_{\perp} \approx 58.6 \mathrm{~m}^{-1}$. If we assume these values to be approximately constant for the present discussion, then equation (2) becomes $n_{0}=1.22 B_{0}-1.25$ (again with $n_{0}$ and $B_{0}$ in units of $\left(1 \times 10^{17} \mathrm{~m}^{-3}\right)$ and $(\mathrm{mT})$, respectively). This is only in moderate agreement with that obtained from the best-fit linear function above. However, equation (2) assumes a uniform magnetic field and density, which is not the case here. Thus some type of average magnetic field and density values would be more appropriate. Using instead an average axial density (found using data in [17]) and an average axial magnetic field, the best-fit linear function is modified to $n_{\text {ave }}=0.98 B_{\text {ave }}-1.11$. This shows fairly reasonable agreement with that found from equation (2), and more importantly, shows that the helicon dispersion relation accounting for finite electron mass (and hence allowing for the existence of a TG mode) has to be used for these low-field peaks. In simple helicon theory electron inertia is ignored, and thus the second term on the left-hand side of equation (1) vanishes. For a given wavenumber this equation is then still 

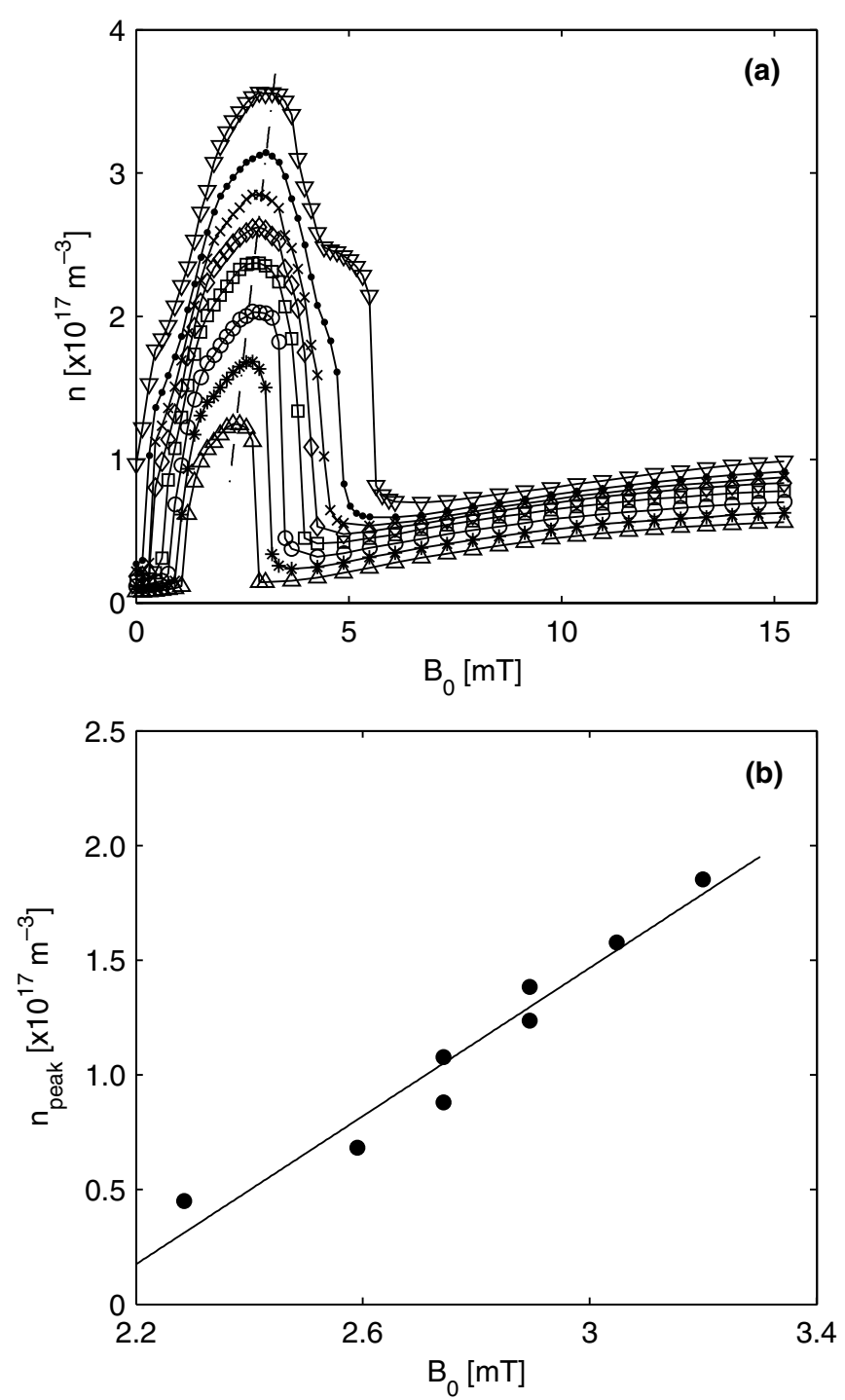

Figure 4. (a) Plasma density within the source region $(z=-10 \mathrm{~cm})$ as a function of the maximum applied magnetic field (as the source coil is varied, with the exhaust coil off), at a number of gas pressures; $0.04 \mathrm{~Pa}$ (open triangles), $0.06 \mathrm{~Pa}$ (stars), $0.08 \mathrm{~Pa}$ (open circles), $0.12 \mathrm{~Pa}$ (open squares), 0.16 $\mathrm{Pa}$ (open diamonds), 0.2 $\mathrm{Pa}$ (crosses), $0.24 \mathrm{~Pa}$ (dots), 0.36 Pa (open upside-down triangles). The straight dashed line illustrates that the peak density is linearly related to the peak magnetic field as the pressure is increased. The rf power input is $250 \mathrm{~W}$. (b) Peak density (corrected for sheath area effects of the LP) as a function of peak magnetic field. The solid line is a best-fit linear function, indicating the proportionality identified in $(a)$.

linear, but is now a straight line passing through the origin. It is clear from the results above though that the best-fit straight line in figure 3 does not pass through the origin. Thus simple helicon theory fails at these low magnetic fields, and the more complicated dispersion relation of equation (1) must be used.

Equation (2) says only that the density and magnetic field should be linearly related, and says nothing about the power input or system pressure. Both these factors affect the plasma density, and thus if the power input is kept constant, similar linear behaviour should be present as the pressure is varied (if we continue to assume that $k_{z}$ and $k_{\perp}$ remain approximately constant, that is). This is shown in figure $4(a)$ for a number of gas pressures, and a power input of $250 \mathrm{~W}$. We again observe similar behaviour to figure $3(a)$, with the density peaks becoming broader and higher as the pressure is increased. We also note that the density at $0.36 \mathrm{~Pa}$ (open upside-down triangles) is almost 3 times larger than that at $0.04 \mathrm{~Pa}$ (open triangles). The low pressure limit for peak formation is observed to be approximately $0.027 \mathrm{~Pa}$ (not shown in figure 4(a)), while a peak was still observed for pressures higher than $0.65 \mathrm{~Pa}$ (also not shown in figure 4(a)). The dashed line in figure 4(a) shows the approximate linear relationship between the density peaks as the pressure is increased. It is interesting to note that a 'shoulder' begins to appear on the right-hand side of the density peaks for pressures above $0.2 \mathrm{~Pa}$, becoming very prominent at $0.36 \mathrm{~Pa}$ for a magnetic field of around $5 \mathrm{mT}$. The appearance of this 'shoulder' might be related to a change in the power deposition process that occurs at higher pressures, although this has not yet been investigated further. In figure $4(b)$ we have plotted the peak density (corrected for sheath area effects of the LP) as a function of the magnetic field at this peak density. A strong linear relationship is again seen between these variables, consistent with the prediction of equation (2). The best-fit line gives $n_{\text {peak }}=1.6 B_{\text {peak }}-3.38$. Since we do not have the density profiles for all of these pressure cases, an average density correction cannot be used; however the coefficients of this equation are of a similar order to that from equation (2). This would indicate that uniform helicon theory might still be used to give reasonable predictions, even if the magnetic field and plasma density are not uniform, although this need not be true in general, especially for systems with a larger degree of nonuniformity.

A number of magnetic field test conditions are then performed on the system. In the first test we reverse the current in the source coil (thus changing the direction of the magnetic field, with magnetic north now pointing towards the pump), while in the second test the magnetic field is ramped down from 16 to $0 \mathrm{mT}$ to check for hysteresis. These tests are performed at a pressure of $0.04 \mathrm{~Pa}$ and an input power of $250 \mathrm{~W}$, and the results are shown in figure 5. For the first test (dashed line in figure 5) it can be seen that a density peak of similar height and width forms for approximately the same magnetic field as for the original field configuration (solid line in figure 5). Double-saddle field antennas (as used here) are not sensitive to the magnetic field direction as they can launch both $m=1$ and $m=-1$ helicon waves. This is in contrast to phased helical antennas, which can preferentially excite $m=1$ or $m=-1$ modes. Consequently, the magnetic field direction has been observed to be important in the formation of density peaks with these antennas [11]. For the second test, after ramping the magnetic field up (solid line), the magnetic field was then ramped down (stars in figure 5). Here the only hysteresis observed occurs at the right-hand boundary of the peak $\left(B_{0} \approx 2.5 \mathrm{mT}\right.$ ), otherwise the system shows virtually identical behaviour (both in shape and magnitude) to that for the original case. This is in contrast to observations in [16] where as the magnetic field was decreased, the plasma density remained high, even with no magnetic field at all. It was argued that once the plasma reaches the high density mode, inductive 


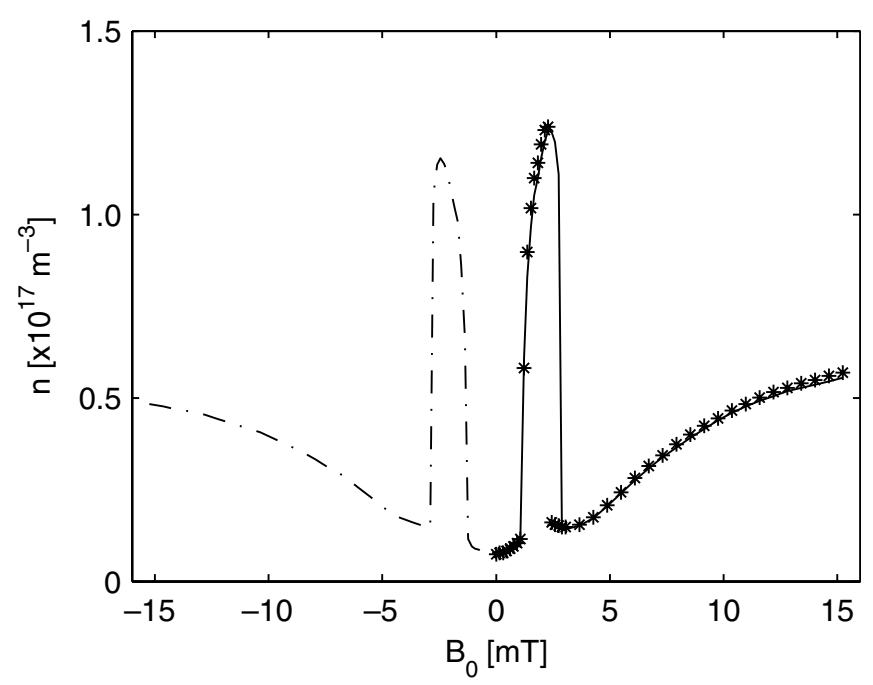

Figure 5. Plasma density within the source region $(z=-10 \mathrm{~cm})$ as a function of the maximum applied magnetic field (as the source coil is varied, with the exhaust coil off), for a number of magnetic field test cases; magnetic field ramped up from $0 \mathrm{mT}$ (solid line), magnetic ramped down from $16 \mathrm{mT}$ (stars), magnetic field direction reversed (magnetic north pointing towards the pump) and ramped up from $0 \mathrm{mT}$ (dashed line). The gas pressure is $0.04 \mathrm{~Pa}$ and the $\mathrm{rf}$ power input is $250 \mathrm{~W}$.

coupling becomes the dominant heating mechanism, and that this can sustain the system even with no applied magnetic field. This does not seem to be the case here, where no hysteresis is seen, suggesting (together with the results of figures 3 and 4) that the density peaks are predominantly due to a wave mode. All of the measurements above have been made with only the source coil operating, and it is of interest to investigate the effect of the exhaust coil. To do this we run a number of tests with just the exhaust coil operating (source coil off) for the low $(0.04 \mathrm{~Pa})$ and high $(0.36 \mathrm{~Pa})$ pressure conditions already investigated in figure $4(a)$. This is shown in figure 6 , together with the corresponding cases from figure 4(a) where only the source coil was operating. At low pressure, no density peak is observed with just the exhaust coil on (open triangles). The plasma remains a dull purple colour, and at no point during the magnetic field ramp does the matching change significantly. By contrast, for the high pressure cases (open circles and open squares), a density peak is observed. It initially shows similar behaviour to that with just the source coil, before levelling off at around $B_{0} \approx 2 \mathrm{mT}$. It then drops off near $B_{0} \approx 3.5 \mathrm{mT}$, well before the corresponding case with just the source coil operating. These measurements were made at an axial location of $z=-10 \mathrm{~cm}$, and it is thus of interest to see how the density profile is affected by the change of magnetic field coils. Figure 7 shows the axial density profiles at $250 \mathrm{~W}$ for a maximum magnetic field of $2.1 \mathrm{mT}$ (that is, for the same current in either the source or exhaust coil). The low pressure case with just the exhaust coil (open triangles) shows a low and relatively constant density of approximately $1 \times 10^{16}$, being slightly higher in the diffusion chamber. The low pressure case with the source coil (open diamonds), however, is about 10 times larger, peaking in the source region at $z=-15 \mathrm{~cm}$ (close to the source coil itself). Similar behaviour is present for

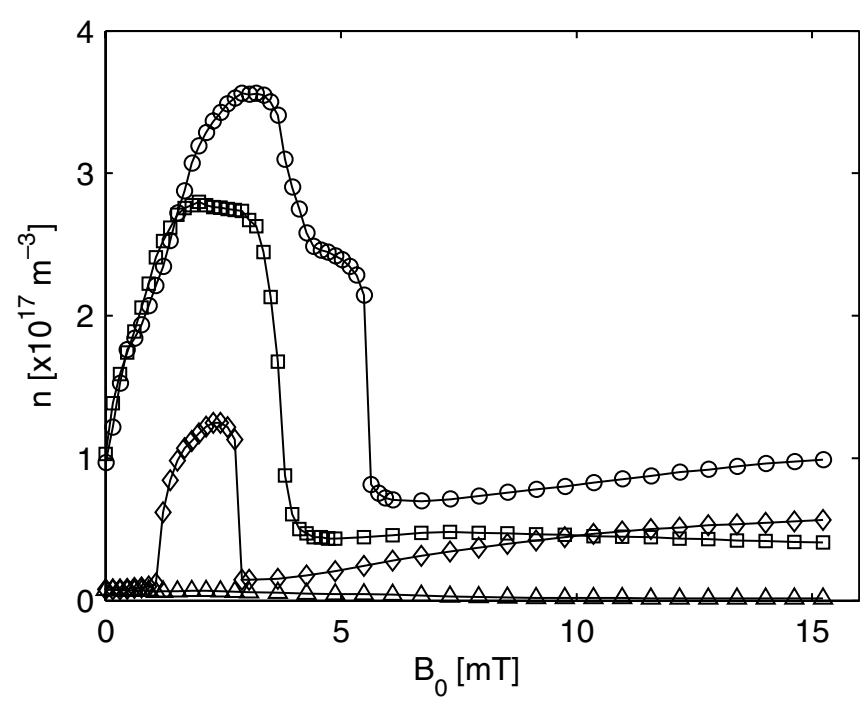

Figure 6. Plasma density within the source region $(z=-10 \mathrm{~cm})$ as a function of the maximum applied magnetic field for a number of tests cases; gas pressure $0.04 \mathrm{~Pa}$ and source coil on with exhaust coil off (open diamonds), gas pressure 0.04 $\mathrm{Pa}$ and exhaust coil on with source coil off (open triangles), gas pressure $0.36 \mathrm{~Pa}$ and source coil on with exhaust coil off (open circles), gas pressure $0.36 \mathrm{~Pa}$ and exhaust coil on with source coil off (open squares). The rf power input is $250 \mathrm{~W}$. Note that no density peak is observed at $0.04 \mathrm{~Pa}$ with just the exhaust coil on (open triangles).

the high pressure case with the source coil (open circles), which has a density about 3 times higher than the low pressure case. The high pressure case peaks at around $z=-15 \mathrm{~cm}$, before rapidly decaying downstream. The high pressure case for the exhaust coil (open squares), however, peaks significantly downstream at around $z=-6 \mathrm{~cm}$, and shows an almost symmetrical profile about this point. In a previous publication [17] the low pressure case for the source coil was shown to approximately follow the magnetic field. We check this for the high pressures cases by plotting the normalized densities, together with their respective normalized magnetic fields in figure 8 . Here we see a strong correlation between the observed density profiles, and the magnetic field profiles. This suggests that the plasma is confined by the magnetic field, and forced to undergo expansion as the field diverges.

\section{2. $R F$ circuit characterization}

With the source characterization complete, the matching network/antenna circuit response is investigated for some of the conditions above. Measurements of the effective antenna loading resistance, $R_{\text {eff }}$, and quality factor, $Q$, of the circuit are taken, using the rf current and high voltage probes. A schematic of the matching network/antenna has been shown previously in figure 2. Here the antenna is assumed to be composed of an effective inductance, $L_{\text {ant }}$, a circuit resistance, $R_{\text {ant }}$ (which includes all ohmic and contact resistances, and any eddy current losses relevant to the antenna and matching network; this resistance is sometimes called the vacuum resistance), as well as a plasma resistance, $R_{\mathrm{p}}$ (which includes all power losses attributed to the plasma, including any capacitive coupling, and inductive or wave coupling). 


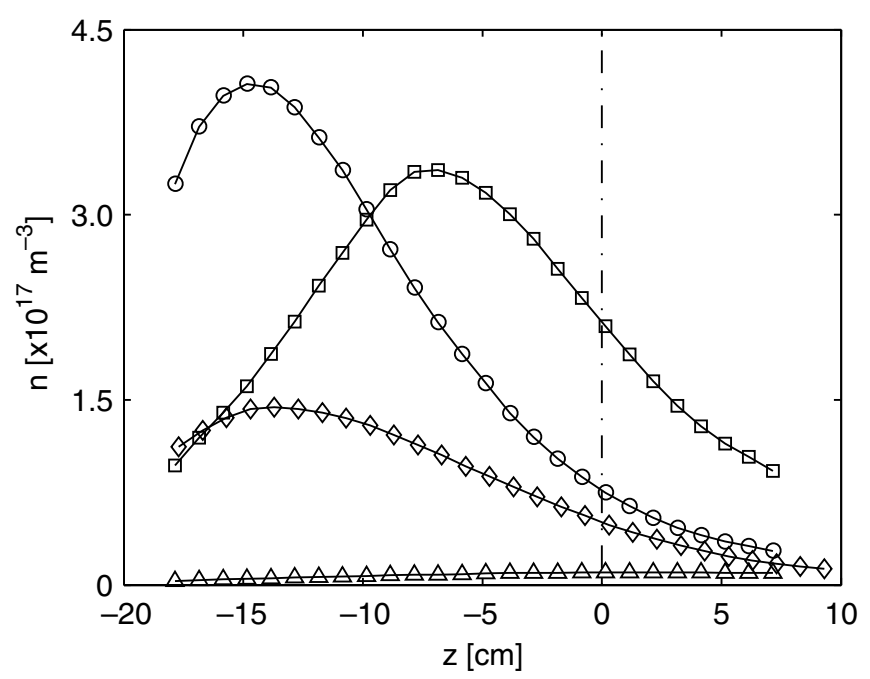

Figure 7. Axial plasma density profiles for the test cases in figure 6; gas pressure $0.04 \mathrm{~Pa}$ and source coil on with exhaust coil off (open diamonds), gas pressure $0.04 \mathrm{~Pa}$ and exhaust coil on with source coil off (open triangles), gas pressure $0.36 \mathrm{~Pa}$ and source coil on with exhaust coil off (open circles), gas pressure $0.36 \mathrm{~Pa}$ and exhaust coil on with source coil off (open squares). The $\mathrm{rf}$ power input is $250 \mathrm{~W}$, and the maximum magnetic field is $2.1 \mathrm{mT}$. The vertical dashed line marks the source tube exit.

Since the antenna/plasma is matched under each measurement condition (both for the measurements in section 4.1, and all subsequent measurements), and the observed reflected power is typically $<1 \%$, all of the power input from the rf generator must then be dissipated in the antenna/plasma system. The current in the antenna is related to the total effective antenna loading resistance, $R_{\mathrm{eff}}=R_{\mathrm{ant}}+R_{\mathrm{p}}$, and the net power flow to the antenna by

$$
P_{\mathrm{f}}-P_{\mathrm{r}}=I_{\mathrm{rms}}^{2}\left(R_{\mathrm{ant}}+R_{\mathrm{p}}\right),
$$

where $P_{\mathrm{f}}$ and $P_{\mathrm{r}}$ are the forward and reflected powers, respectively, and $I_{\mathrm{rms}}=I_{\mathrm{rf}} / \sqrt{2}$, with $I_{\mathrm{rf}}$ being the amplitude of the sinusoidally varying current. Using the current probe, $I_{\text {rms }}$ can be determined, and using the known power output from the generator (previously calibrated with a $50 \Omega$ resistive load), $R_{\text {eff }}$ can be determined from equation (5). Using this approach we measure the effective loading resistance as a function of the maximum applied magnetic field for a number of rf powers, as shown in figure $9(a)$. The gas pressure is kept constant at $0.04 \mathrm{~Pa}$. As is seen, distinct resistance peaks are present for powers above approximately $100 \mathrm{~W}$, well correlated with the density peaks in figure 3(a). While the peaks appear 'sharper' than those of the density peaks, they are of a similar width. It is interesting to note that the peak resistance is approximately linearly related to the magnetic field at the peak resistance, as demonstrated by the straight dashed line in figure $9(a)$. The quality factor, $Q$, of a resonant circuit (such as the matching network/antenna system) represents a measure of the stored to dissipated energy within the circuit, and is related to the effective antenna loading resistance. A low $Q$ factor means the effective loading resistance of the circuit has increased, and thus affords a second independent check of the measurements made with the rf current probe. The $Q$ factor can be shown to

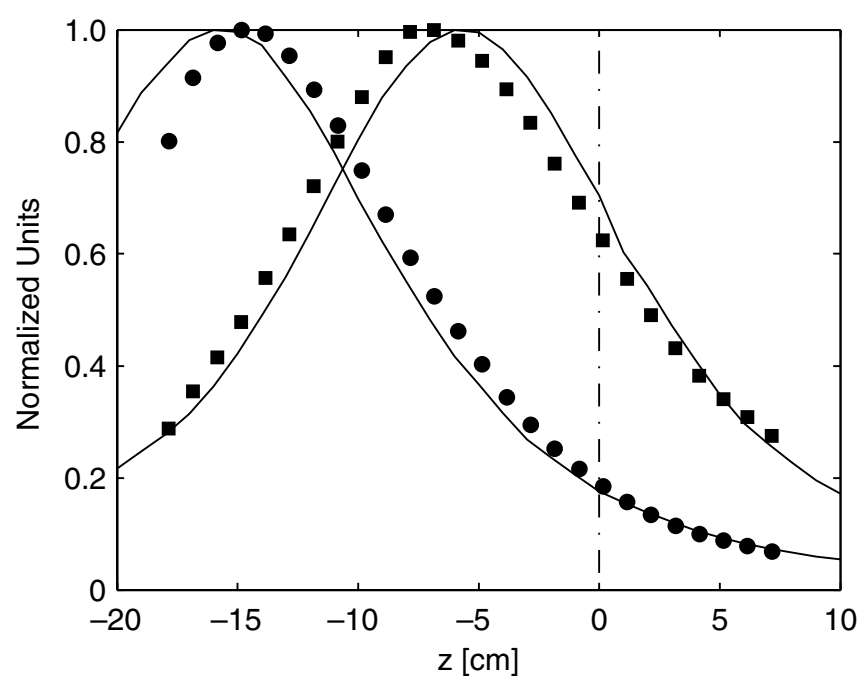

Figure 8. Normalized axial plasma density profiles for the high pressure, $0.36 \mathrm{~Pa}$, test cases in figure 7 ; source coil on and exhaust coil off (closed circles), exhaust coil on with source coil off (closed squares). The solid lines are normalized axial magnetic field profiles with the source coil on (exhaust coil off; left curve) and the exhaust coil on (source coil off; right curve). The rf power input is $250 \mathrm{~W}$, and the maximum magnetic field is $2.1 \mathrm{mT}$. The vertical dashed line marks the source tube exit.

be equal to the ratio of voltages across the antenna, and from figure 2 is given by

$$
Q=\frac{V_{\mathrm{B}}}{V_{\mathrm{A}}},
$$

where $V_{\mathrm{A}}$ and $V_{\mathrm{B}}$ are the rf voltages measured at points $\mathrm{A}$ and $\mathrm{B}$ in figure 2 , respectively. $Q$ factor measurements are shown in figure $9(b)$, where it is seen that they very closely mirror those of the resistance measurements. The $Q$ factor begins at approximately 10-11 for fields below $1 \mathrm{mT}$, before rapidly dropping to a minimum of less than 7 at around $2.5 \mathrm{mT}$. This decrease in $Q$ implies an increased circuit resistance, consistent with the measurements in figure $9(a)$. Again an almost linear relationship is seen between the minimum $Q$ factors and the magnetic field at these minima. The power transfer efficiency, $\eta$, is a measure of how much of the input power from the generator is absorbed in the plasma, and can be given by

$$
\eta=\frac{P_{\mathrm{abs}}}{P_{0}}=\frac{R_{\mathrm{p}}}{R_{\mathrm{ant}}+R_{\mathrm{p}}},
$$

where $P_{\text {abs }}$ is the total absorbed power by the plasma and $P_{0}$ is the total power input from the generator. The external circuit resistance, $R_{\text {ant }}$, can be determined from equation (5) when operating with no plasma in the reactor (this then means that if the system is matched, all of the input power must be dissipated in the matching network/antenna system). This gives a value of $R_{\text {ant }} \approx 0.21 \Omega$. Measurements of a similar system (but with a slightly longer antenna of $18 \mathrm{~cm}$ so that a larger resistance would be expected) [25] have yielded values of the external resistance of $0.3 \Omega$, close to our value giving us confidence in its magnitude. With the external resistance known, we calculate the power transfer efficiency as a function of total power input for the peak resistances in figure $9(a)$. This is shown in figure $10(a)$. Here it is seen that the power transfer 

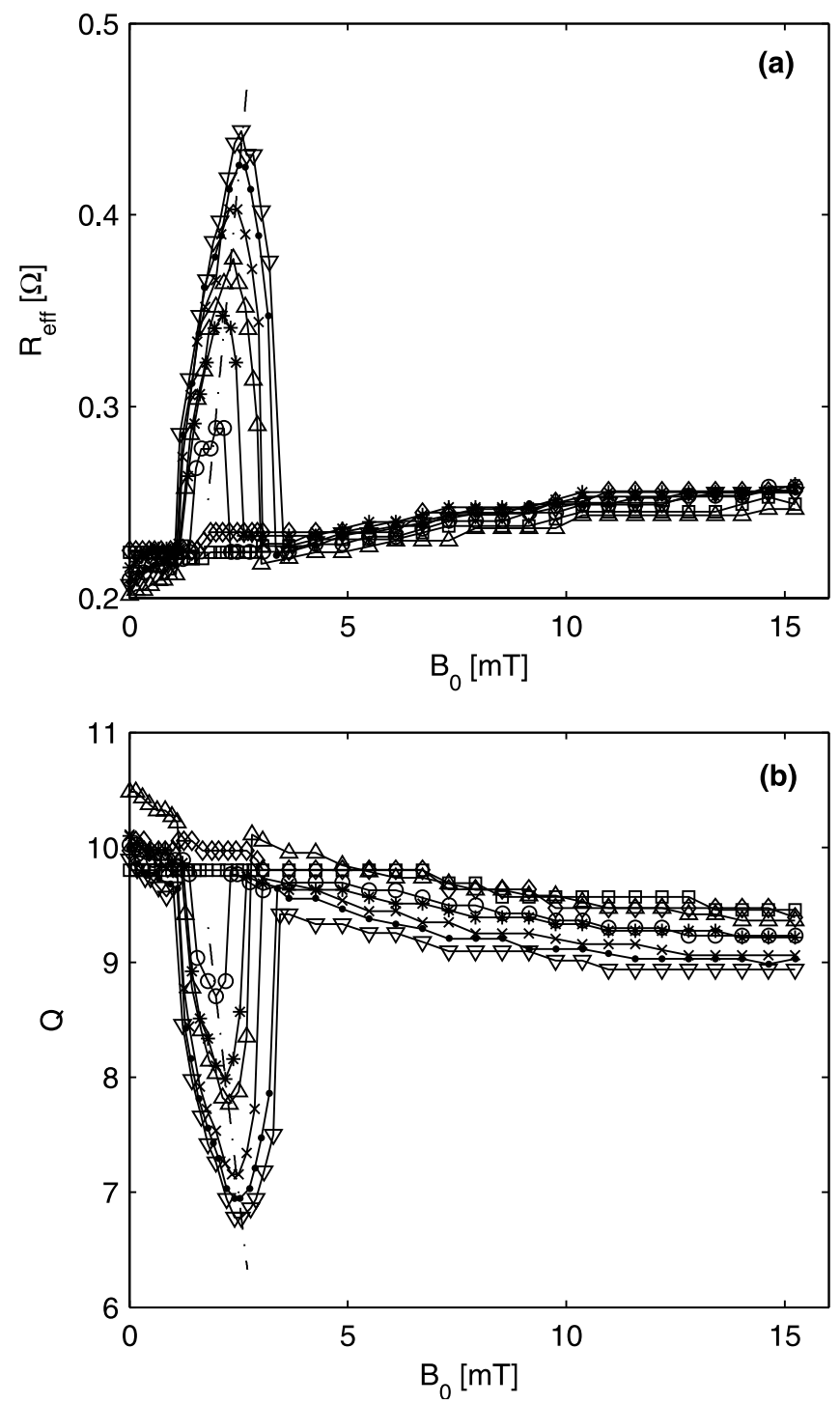

Figure 9. (a) Effective antenna loading resistance as a function of the maximum applied magnetic field (as the source coil is varied, with the exhaust coil off), at a number of rf powers; $50 \mathrm{~W}$ (open squares), $100 \mathrm{~W}$ (open diamonds), $150 \mathrm{~W}$ (open circles), $200 \mathrm{~W}$ (stars), $250 \mathrm{~W}$ (open triangles), $300 \mathrm{~W}$ (crosses), $350 \mathrm{~W}$ (dots), $400 \mathrm{~W}$ (open upside-down triangles). The straight dashed line illustrates that the peak resistance is linearly related to the peak magnetic field as the power is increased. The gas pressures is $0.04 \mathrm{~Pa}$. (b) Quality factor, $Q$, as a function of maximum applied magnetic field for the same rf powers as in $(a)$. Again, the straight dashed line illustrates that the minimum $Q$ factor is linearly related to the peak magnetic field.

efficiency is initially quite low, less than $30 \%$ for low powers, but then rises rapidly, before levelling off at around $50 \%$ for an input power of approximately $400 \mathrm{~W}$. Figure $10(b)$ shows the absorbed power plotted as a function of the magnetic field at the maximum efficiency points in $R_{\text {eff }}$ and $Q$ of figure 9 . Here we again see an approximately linear relationship, similar to figure $3(b)$.

Similar measurements to figure 9 are then taken for a number of gas pressures, while keeping the rf power constant at $250 \mathrm{~W}$. Both the resistances and $Q$ factors are shown in figure 11 . Once again the resistance starts out quite low
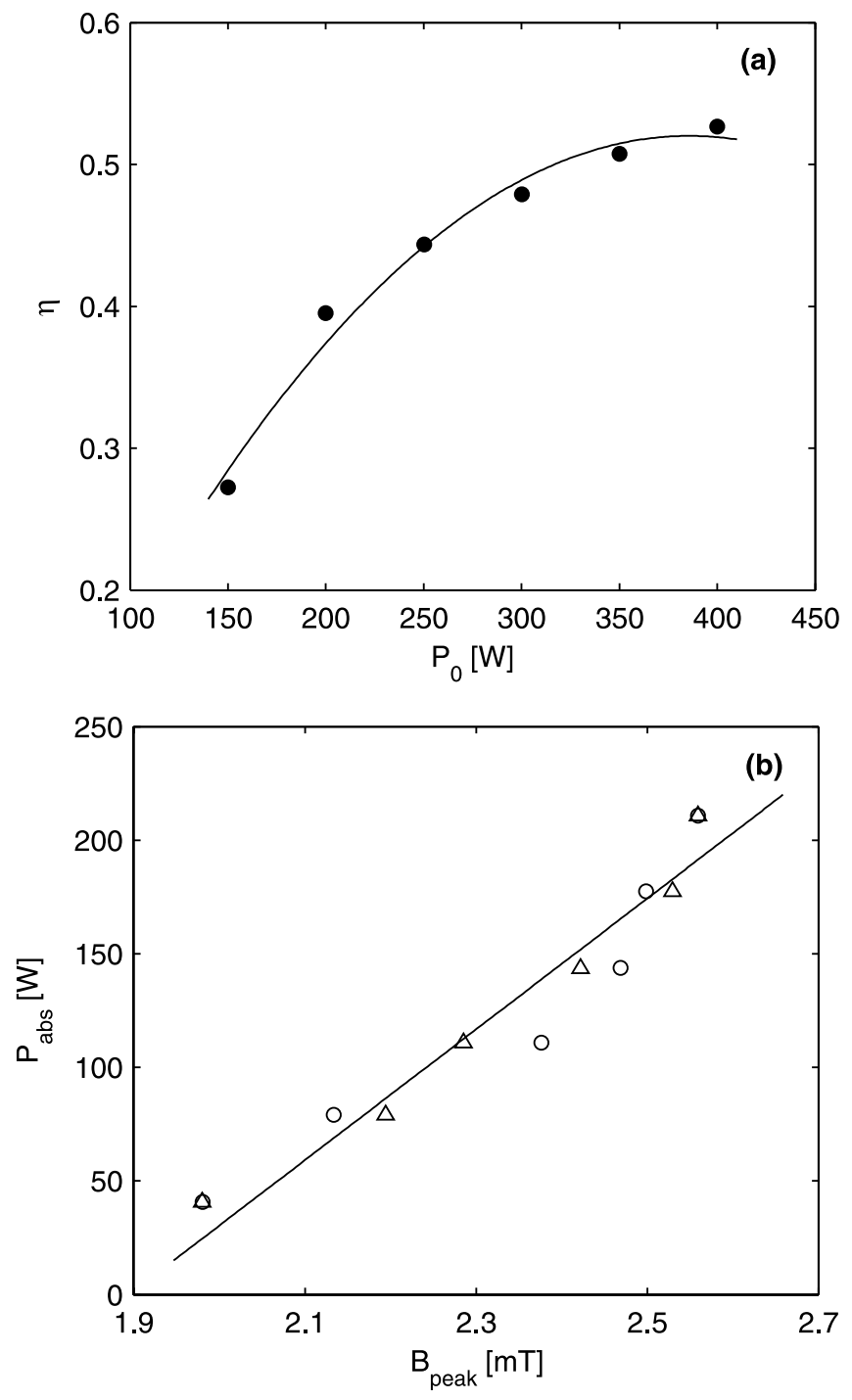

Figure 10. (a) Power transfer efficiency, $\eta$, as a function of total applied $\mathrm{rf}$ power using the resistances from figure $9(a)$. The solid line serves as a visualization aid. $(b)$ Absorbed power as a function of the peak magnetic field, with peaks from figure $9(a)$ (open circles), and peaks from figure $9(b)$ (open triangles). The solid line shows a best-fit linear function to the data.

$(\approx 0.2 \Omega)$, before increasing to around $0.55 \Omega$. The peaks are also well correlated with those of the density measurements in figure 4. The $Q$ factors, mirroring the resistance results, start high at around 10, before decreasing to around 6 at about $3 \mathrm{mT}$. By contrast to the results in figure 9 however, both the resistances and $Q$ factors eventually level off at a certain value. This is more clearly seen in figure $12(a)$ where the power transfer efficiency has been plotted as a function of gas pressure. The efficiency initially increases rapidly from just above $30 \%$, before flattening off at just above $60 \%$. This suggests that even though the density increases (see figure $4(a)$ ), the power absorbed by the plasma no longer increases. Figure 12(b) shows the magnetic field at the maxima/minima of figure 11 for the resistance and $Q$ factor measurements. As is seen, the $Q$ factors are a minimum at approximately the same location that the resistances are a maximum, thus showing good consistency between measurements. 

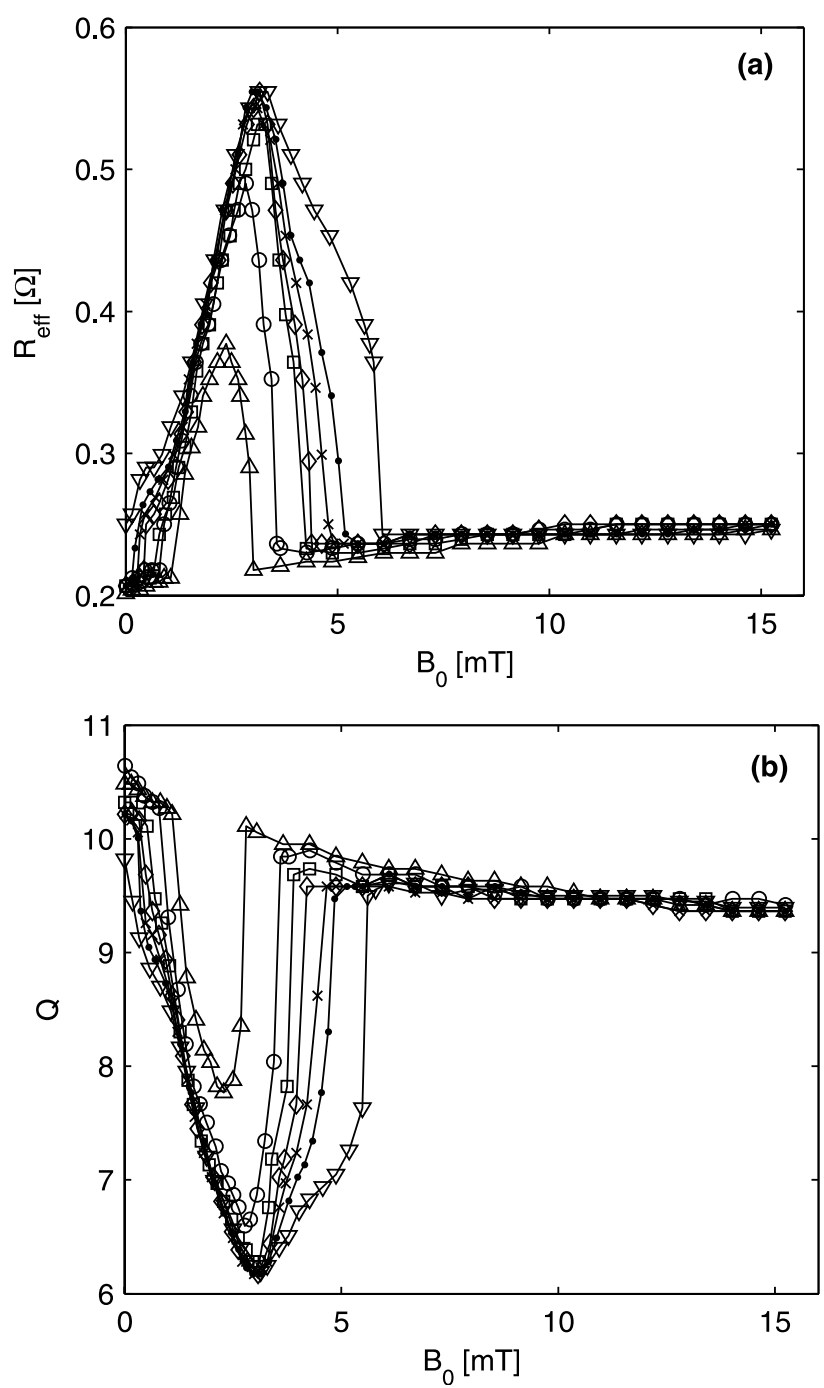

Figure 11. (a) Effective antenna loading resistance as a function of the maximum applied magnetic field (as the source coil is varied, with the exhaust coil off), at a number of gas pressures; $0.04 \mathrm{~Pa}$ (open triangles), $0.08 \mathrm{~Pa}$ (open circles), $0.12 \mathrm{~Pa}$ (open squares), $0.16 \mathrm{~Pa}$ (open diamonds), 0.20 Pa (crosses), 0.24 Pa (dots), 0.36 Pa (open upside-down triangles). The rf power input is $250 \mathrm{~W}$. (b) Quality factor, $Q$, as a function of the maximum applied magnetic field for the same gas pressures as in $(a)$.

We note that the resistances in figures 9 and 11, after accounting for the antenna resistance of $0.21 \Omega$, give plasma resistances between 0 and $0.35 \Omega$, which while quite low, are similar to values measured by Degeling [30] $(0-0.6 \Omega)$ for helicon waves in magnetic fields from 20 to $150 \mathrm{G}$, as well as those from a computational study by Cho [19], where resistances between 0 and $1 \Omega$ were obtained for similar conditions (although this computational work assumes a uniform magnetic field and plasma density). The capping of the power transfer efficiency in figures 10 and 12 is similar to other work on helicon systems [25,31].

\section{Discussion}

In the results presented in section 4 , it was noted that the observed density peaks became both higher and wider as the power or pressure was increased. This was correlated with
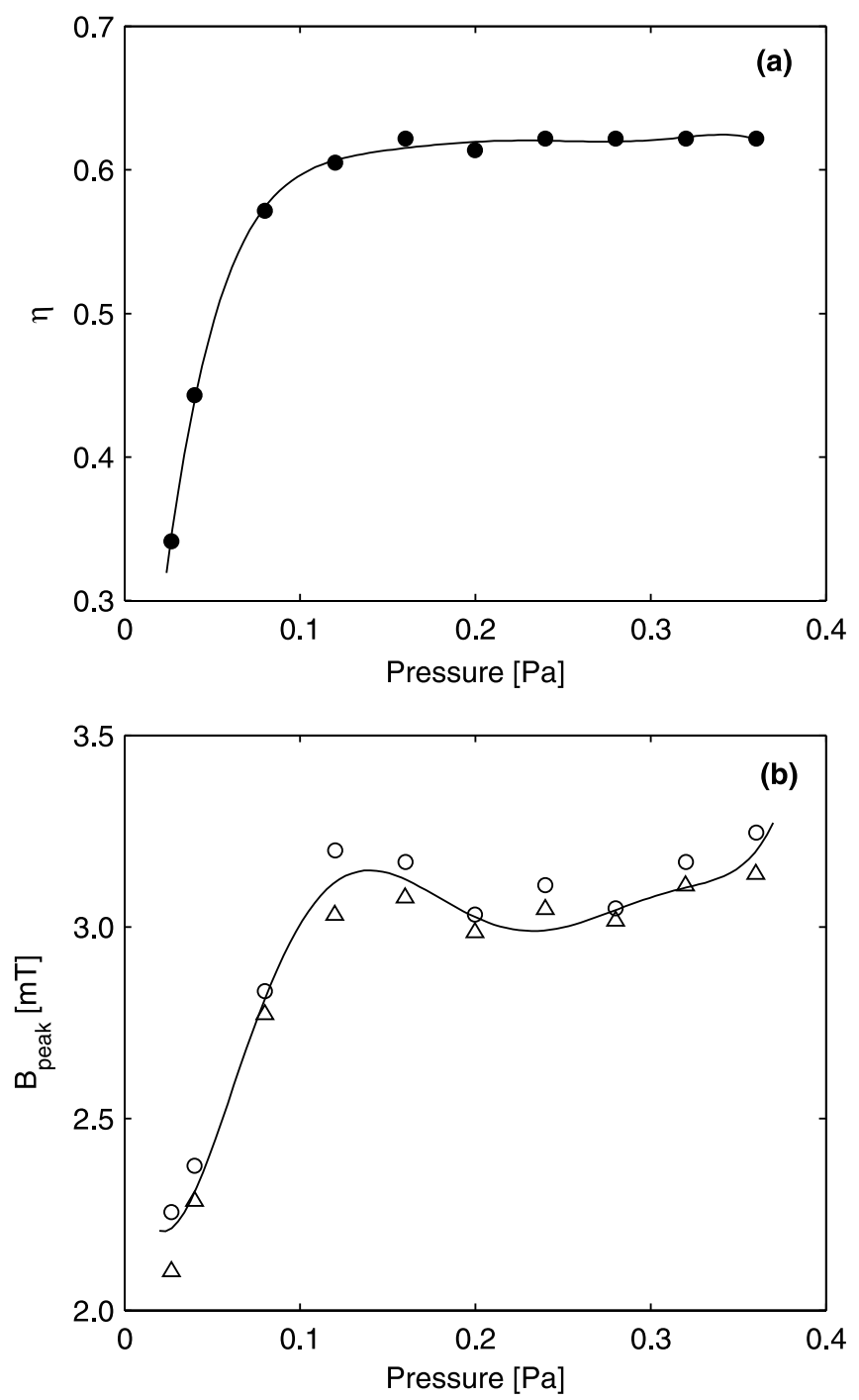

Figure 12. (a) Power transfer efficiency, $\eta$, as a function of gas pressure using the resistances from figure $11(a)$. The solid line serves as a visualization aid. (b) Peak magnetic fields as a function of gas pressure, with peaks from figure 11( $a$ ) (open circles), and minima from figure 11(b) (open triangles). The solid line serves as a visualization aid.

similar behaviour in the measured circuit resistance. The increased resistance offers an explanation as to why the plasma density is able to increase by such large amounts as the magnetic field is changed, since increased resistance means a larger percentage of the input power is actually deposited within the plasma. From a simple particle balance [24], the electron temperature depends only on the system geometry and neutral gas pressure, and is independent of the power input. Thus the Bohm velocity $\left(u_{\mathrm{B}}\right)$, and the total effective energy carried away per electron-ion pair lost at the walls ( $\left.E_{\text {eff }}\right)$, would be expected to be constant [24]. Thus from a power balance [24], as the power increases, the density must increase linearly, or more specifically, the increased density is a complete result of the increased absorbed power. However, when the input power is kept constant, and the pressure is changed, the electron temperature (and hence $u_{\mathrm{B}}$ and $E_{\text {eff }}$ [24]), changes, and thus so too does the power flux to the source tube boundaries. Now, the increased density is not a sole result 
of an increased absorbed power. This is more clearly seen from figure 12, where the power transfer efficiency (and hence total absorbed power) levels off, becoming almost constant, suggesting that no further 'extra' power is deposited within the plasma, yet from figure 4 the density is still seen to rise.

We note from the results in section 4 that the resistance peaks occur approximately at the same location as the density peaks. As pointed out by Cho [19], this is expected to be approximately true if $R_{\mathrm{p}}$ is not significantly larger than $R_{\text {ant }}$. As seen from section 4.2, the plasma resistance is of a similar magnitude to the circuit resistance, consistent with this view. Since minimal hysteresis effects were observed (see figure 5), and given the previous wave measurements made in the present reactor [22], it would seem as if power is deposited predominately by the wave mode. Thus the measured resistance peaks in section 4.2 seem likely to be a result of increased plasma resistance due to wave coupling, as opposed to some type of enhanced capacitive coupling or transformer action associated with simple inductive coupling. This means that we can gain insight into what is happening by looking at the numerical results within the literature $[18,19]$ (which ignore capacitive coupling effects). The numerical results suggest that for a fixed geometry, $R_{\mathrm{p}}$ is a strong function of $n_{0}$ or $B_{0}$ (this is of course not surprising, as these factors change the plasma dielectric, and hence impact wave propagation significantly). For a given initial plasma condition (say at a certain point located on one of the density peaks in figures $3(a)$ or $4(a))$, if the magnetic field is instantaneously increased, the plasma dielectric will also change, and this could act to increase $R_{\mathrm{p}}$ for example, and hence the power transfer efficiency. This then leads to a larger absorbed power which consequently increases the plasma density. This increased density then allows the plasma resistance to increase further (for example), which then increases the absorbed power again, and so on, until some type of new stable equilibrium point is reached (say another point on one of the density peaks in figures 3(a) or 4(a) adjacent to the initial starting point). Thus a feedback mechanism would exist within the system. Then as $B_{0}$ is increased further, this process can repeat. As the total $\mathrm{rf}$ power input from the generator or gas pressure is increased, it is perhaps not hard to imagine that the system density would be slightly higher before the magnetic field is instantaneously changed for the first time, so that the wave mode can be initiated (lost) at an earlier (later) magnetic field (in order to still satisfy the dispersion relation, as discussed in section 4.1 ), hence the broadening of the density peaks with increased power or pressure.

The above process of course relies on there being a mechanism by which the waves can interact with the plasma, and hence transfer power to the electrons. In helicon systems in general the power transfer process is complicated, with a number of different mechanisms proposed; wave-particle trapping in the parallel component of the helicon wave [15, 30], heating of electrons by electrostatic Trivelpiece-Gould (TG) modes [32] (leading to helicon wave damping due to mode coupling), and parametric decay of helicon modes into ion acoustic and TG modes [33]. Cho [19] shows that the antenna resistance is large when the helicon and TG waves merge $\left(k_{\perp_{1}}=k_{\perp_{2}}\right.$ ), suggesting that mode coupling could be playing a role in the formation of these low-field peaks. Under these conditions, for a constant axial wavenumber $k_{z}$, the peak density is expected to be quadratically related to the peak magnetic field $\left(n_{0} \propto B_{0}^{2}\right)$ [19], a scaling which is not seen in figures 3 and 4 . While it should be said that this scaling cannot be ruled out (since the experimental densities span a fairly narrow range), Cho [19] also discusses that this quadratic scaling is not accurate over a wide density range, since as the density changes the region of maximum resistance deviates from the mode coupling region (depending on the density). In addition, we have recently shown [22] that in the diverging fields presented here, the magnetic field decays to values giving electron cyclotron frequencies close to the wave frequency near the source tube exit, thus suggesting dopplershifted cyclotron resonance (DSCR) as a possible additional mechanism enhancing the heating process; a process that previous computational codes $[18,19]$ would not account for. In that study [22], during the density peaks of figure 4(a) (for a pressure of $0.08 \mathrm{~Pa}$ ) wave behaviour was observed in the source region, but not in the downstream region. However, as the magnetic field was increased slightly near the source exit (which was suggested to decrease the effectiveness of DSCR [22]) waves were observed to 'escape' into the downstream region. This resulted in a decrease in source density and an increase in the downstream density, consistent with the view that waves escaping from the source region can deposit their power into the downstream chamber. However, as the magnetic field was increased further, the density in the source region eventually became too low, and the wave mode was lost (yielding plasma behaviour similar to that occurring just before and after the density peaks shown in section 4.1). In the present case, as the magnetic field is increased, the cyclotron resonance region (located at a magnetic field of approximately $4.8 \mathrm{mT}$ ) would shift further downstream, and thus if it moves sufficiently far, waves could escape into the downstream region, and the density in the source might decrease, until eventually the mode is lost altogether. This could serve as the upper (right-hand side) boundary of the density peaks seen in section 4.1. It is interesting to consider what might cause the lower (left-hand side) boundary. From classical helicon theory [3] right-hand polarized waves (of which the helicon is one) will not propagate past a cyclotron resonance point (or more specifically in the region where $\omega_{\mathrm{ce}}<\omega<\omega_{\mathrm{pe}}$ ). Initially the magnetic field is low, and since it is diverging, there is a very small 'cavity' region (bounded by the cyclotron resonance points in the magnetic field profile) permitting wave transmission. As the field is increased, this region increases in width, until eventually a wavelength might fit within this cavity. Since the antenna has a length of $10 \mathrm{~cm}$, helicon waves with a wavelength of about $20 \mathrm{~cm}$ would most strongly be excited $[2,24]$ in the present system. Thus the lower (left-hand side) boundary of the density peaks in section 4.1 might be initiated when the 'cavity' is sufficiently wide that a wavelength can fit within it. Observation of figure 4 shows that at very high pressures $(0.36 \mathrm{~Pa})$ the density at low fields $\left(B_{0}<1 \mathrm{mT}\right)$ is still high (even with no magnetic field). Since a magnetic field is required for helicons to propagate, this suggests that 
inductive coupling might begin to become significant at these low fields and high pressures, before the true wave mode has been initiated.

It would appear based on the results in section 4 that despite the nonuniformities in the magnetic field and plasma density profiles, the experimental results still follow uniform helicon theory fairly well (an observation also noted in [7]). Figure 8 shows that the density very closely follows the magnetic field, thus the ratio $B / n$ will be approximately constant. From simple helicon dispersion relations [3] this suggests that the plasma refractive index will also be constant, so that helicon waves would 'see' a uniform plasma. For low magnetic fields this is of course not entirely true, since equation (1) cannot be written solely in terms of $B_{0} / n_{0}$ (due to the correction factor accounting for finite electron mass), but the general argument remains the same. Thus even in the presence of nonuniformities in the density and magnetic field profiles, helicon theory can still be used to make reasonable predictions (as long as the nonuniformities are not too severe).

\section{Conclusions}

In summary, we have performed a detailed characterization of a helicon plasma source in the presence of a low, diverging magnetic field. The results show that despite the nonuniformities within the plasma density and magnetic field profiles, uniform helicon theory still holds reasonably well. No significant hysteresis effects were observed in these lowfield peaks, and it is found that the axial plasma density profiles very closely follow that of the applied magnetic field. Electrical measurements within the matching network/antenna show local peaks in the plasma resistance well correlated with the observed density peaks, indicating that the power transfer efficiency between the antenna and plasma increases.

\section{References}

[1] Boswell R W 1970 Phys. Lett. A 33457

[2] Boswell R W 1984 Plasma Phys. Control. Fusion 261147

[3] Boswell R W and Chen F F 1997 IEEE Trans. Plasma Sci. 251229

[4] Chen F F and Boswell R W 1997 IEEE Trans. Plasma Sci. 251245
[5] Ellingboe A R and Boswell R W 1996 Phys. Plasmas 32797

[6] Perry A, Conway G, Boswell R W and Persing H 2002 Phys. Plasmas 93171

[7] Chen F F 2008 IEEE Trans. Plasma Sci. 362095

[8] Chen F F, Jiang X and Evans J D 2000 J. Vac. Sci. Technol. A 182108

[9] Chen F F and Torreblanca H 2009 Phys. Plasmas 16057102

[10] Carter C and Khachan J 1999 Plasma Sources Sci. Technol. 8432

[11] Sato G, Oohara W and Hatakeyama R 2007 Plasma Sources Sci. Technol. 16734

[12] Chen F F 1992 J. Vac. Sci. Technol. A 101389

[13] Shinohara S and Soejima T 1998 Plasma Phys. Control. Fusion 402081

[14] Wang S J, Kwak J G, Kim C B and Kim S K 2003 Phys. Lett. A 313278

[15] Degeling A W, Jung C O, Boswell R W and Ellingboe A R 1996 Phys. Plasmas 32788

[16] Lho T, Hershkowitz N, Miller J, Steer W and Kim G H 1998 Phys. Plasmas 53135

[17] Lafleur T, Charles C and Boswell R W 2010 Phys. Plasmas 17043505

[18] Chen F F 2003 Phys. Plasmas 102586

[19] Cho S 2006 Phys. Plasmas 13033504

[20] Charles C and Boswell R W 2004 Phys. Plasmas 111706

[21] Charles C and Boswell R W 2007 Appl. Phys. Lett. 91201505

[22] Lafleur T, Charles C and Boswell R W 2010 Phys. Plasmas 17073508

[23] Takahashi K, Charles C, Boswell R W, Kaneko T and Hatakeyama R 2007 Phys. Plasmas 14114503

[24] Lieberman M A and Lichtenberg A J 2005 Principles of Plasma Discharges and Materials Processing (New York: Wiley)

[25] Chi K K, Sheridan T E and Boswell R W 1999 Plasma Sources Sci. Technol. 8421

[26] Takahashi K, Charles C, Boswell R W and Hatakeyama R 2008 Phys. Plasmas 15074505

[27] Sheridan T E 2000 Phys. Plasmas 73084

[28] Sheridan T E 2010 J. Phys. D: Appl. Phys. 43105204

[29] Lee D and Hershkowitz N 2007 Phys. Plasmas 14033507

[30] Degeling A W 1999 PhD Thesis Australian National University

[31] Shoji T, Sakawa Y, Nakazawa S, Kadota K and Sato T 1993 Plasma Sources Sci. Technol. 25

[32] Shamrai K P and Taranov V B 1996 Plasma Sources Sci. Technol. 5474

[33] Lorenz B, Krämer M, Selenin V L and Aliev Y M 2005 Plasma Sources Sci. Technol. 14623 